In order to optimise the opt out policy, and thereby successful interruption of HIV transmission, interventions to motivate "optouters" should be studied, since the few clients still refusing an HIV test were linked to higher risk behaviour.

\section{P1-S6.18 CHARACTERISTICS OF MEN WHO SEEK RESCREENING FOR STIS AFTER ONCE USING THE HTTP://WWW.IWANTTHEKIT.ORG SCREENING PROGRAM}

doi:10.1136/sextrans-2011-050108.242

${ }^{1} \mathrm{Y}$ H Hsieh, ${ }^{1} \mathrm{M}$ Barnes, ${ }^{1} \mathrm{M}$ Jett-Goheen, ${ }^{1} \mathrm{~N}$ Quinn, ${ }^{1} \mathrm{P}$ Agreda, ${ }^{2} \mathrm{P}$ Whittle, ${ }^{1} \mathrm{~T}$ Hogan, ${ }^{1} \mathrm{C}$ Gaydos. 'Johns Hopkins University, Baltimore, USA; ${ }^{2}$ Baltimore City Health Department, USA

Background The iwantthekit (IWTK) Internet screening program offered an opportunity to study characteristics of men who seek rescreening, as well as determine reported infected status at the previous screening.

Methods We determined characteristics of male repeat users from questionnaires. Predictors of repeat users were identified in a matched case-control study by conditional logistic regression analysis. A case was defined as reporting ever having used IWTK before. A control was a user who reported never using the program before. Two controls were systematically sampled for each case by matching the date of use IWTK of the case within 3 months.

Results During 2007-2010, 115 (14\%) of 852 men who used IWTK for STI testing, indicated that they had used IWTK previously. Among them, $43 \%$ used it $>2$ times. Mean age was $25.8 \mathrm{yr} \pm 8.5 \mathrm{yr}$, and $90 \%$ were currently sexually active. $17 \%$ reported having sex with a male; $35 \%$ had $>5$ partners in the past yr; $58 \%$ had new partners in last 3 months; $49 \%$ currently were having sex $>1$ person. $63 \%$ had been treated for an STI: chlamydia (CT)(74\%), trichomonas (TV)(42\%) and gonorrhoea (GC)(23\%); 6 reported being treated for HIV. By matching time of enrolment, 230 controls were selected. In the multivariate analysis, repeat IWTK users were more likely to be $<30$ years $(\mathrm{OR}=2.04,95 \% \mathrm{CI} 1.04$ to 4.02$)$, have health insurance $(\mathrm{OR}=2.01,95 \% \mathrm{CI} 1.10$ to 3.69$)$, reported ever being tested for an STI (OR=2.01, 95\% CI 1.02 to 3.97), ever been treated for an STI (OR=2.20, 95\% CI 1.14 to 4.23), particularly TV $(\mathrm{OR}=5.16,95 \%$ CI 1.80 to 14.81$)$, and less likely to have penile discharge currently $(\mathrm{OR}=0.24,95 \%$ CI 0.08 to 0.76$)$. Of male repeaters, $80.9 \%$ reported previous test results from IWTK as negative and 22/115 (19.1\%) reported previous result positive-11 had CT, 4 had GC, 8 had TV; 2 of these were mixed infections. At present test, 24 tested positive -19 had CT, 3 had GC, 5 had TV; 3 were mixed infections. Reported CT positivity in last test was associated with current CT test positivity $(p<0.05)$.

Conclusions The Internet screening program IWTK attracted a number of previous male users of IWTK, who practiced high-risk sexual behaviours, to use the program for repeat STI testing. IWTK provided an alternate approach for rescreening previously infected men as well as men reporting high-risk behaviours.

\section{P1-S6.19 COMPARING TWO METHODS OF ESTIMATING CHLAMYDIA SCREENING COVERAGE IN AN URBAN NEIGHBOURHOOD, 2009}

doi:10.1136/sextrans-2011-050108.243

${ }^{1} \mathrm{M}$ Nelson, ${ }^{2} \mathrm{P}$ Pathela, ${ }^{2} \mathrm{~S}$ Blank, ${ }^{2} \mathrm{~J}$ Schillinger. ${ }^{1}$ Cicatelli Associates, Inc, New York, USA; ${ }^{2}$ New York City Department of Health and Mental Hygiene, New York, USA

Background CDC recommends sexually active females aged $<26$ be screened annually for Chlamydia trachomatis (Ct). Only Ct cases are reported to local health departments. Screening coverage estimates, defined as the proportion of the sexually active population tested for $\mathrm{Ct}$, are not routinely available. Without such measures it is difficult to interpret increases in Ct case reports.

Methods We compared 2 approaches to estimating screening coverage in the New York City neighbourhood of Central Brooklyn (CB) in 2009: The "indirect method" used public health surveillance data, and "back calculated" to get the number of sexually active females that must have been screened to yield the number of reported $\mathrm{Ct}$ cases in CB females aged $15-19$ and 20-25 years. Data inputs included: reported number of females with $\geq 1 \mathrm{Ct}$ case in 2009 (730 cases aged $15-19,619$ cases aged 20-25) population estimates (12 772 aged 15-19, 14024 aged 20-25), proportion ever had sex (35\% aged 15-19), proportion sexually active in last 12 months (76\% aged $20-25)$, and Ct positivity (20\% aged $15-19$, $8 \%$ aged $20-25)$. The "direct method" used electronic health record (EHR) data from 8 primary care provider practices in $\mathrm{CB}$ and adjoining zip codes using a common EHR for $>1$ year. EHR data were analysed to determine: numbers of unduplicated female clients aged 15-19 and 20-25, proportion sexually active, and number of sexually active females screened for Ct. The sexually active population was measured in 2 ways; group 1 was defined as females that reported ever having sex (18\% (246/1340) aged $15-19$ and $12 \%$ (302/2419) aged 20-25). Group 2 was defined as females meeting $>1$ of: reported ever having sex; ever prescribed an oral contraceptive by the practice; ever had an STD; ever diagnosed with STD by the practice; ever pap ordered by the practice (38\% (514/1340) aged 15-19 and 38\% (910/2419) aged 20-25).

Results See Abstract P1-S6.19 table 1.

Abstract P1-S6.19 Table 1 Estimated Chlamydia Screening Coverage for Central Brooklyn Females by Method and Age Group, 2009

\begin{tabular}{llllll}
\hline Method & $\begin{array}{l}\text { Age } \\
\text { group }\end{array}$ & $\begin{array}{l}\text { Female } \\
\text { population }\end{array}$ & $\begin{array}{l}\text { Percent } \\
\text { sexually } \\
\text { active }\end{array}$ & $\begin{array}{l}\text { Number } \\
\text { Ct tested }\end{array}$ & $\begin{array}{l}\text { Estimated } \\
\text { screening } \\
\text { coverage }\end{array}$ \\
\hline Indirect & $15-19$ & 12772 & $35 \%(4406)$ & 3632 & $82 \%$ \\
& $20-25$ & 14024 & $76 \%(10630)$ & 8234 & $77 \%$ \\
EHR & & & & & \\
Group 1 & $15-19$ & 1340 & $18 \%(246)$ & 167 & $68 \%$ \\
& $20-25$ & 2419 & $12 \%(302)$ & 178 & $59 \%$ \\
Group 2 & $15-19$ & 1340 & $38 \%(514)$ & 315 & $61 \%$ \\
& $20-25$ & 2419 & $38 \%(910)$ & 518 & $57 \%$ \\
\hline
\end{tabular}

Conclusion The indirect approach yielded a higher Ct screening coverage estimate than the direct approach. By both methods, screening coverage was higher in the $15-19$ age group than the 20-25 age group. For the direct approach, definitions of sexually active women affected measures of screening coverage, particularly for 15-19 year olds. The indirect method can be easily replicated with limited resources. The direct method requires more resources and is contingent on consistent and accurate provider documentation of sexual activity.

\section{P1-S6.20 PERINATAL SCREENING FOR STIS IN THE USA: ADHERENCE TO PREVENTIVE SCREENING RECOMMENDATIONS FOR HIV AND SYPHILIS AMONG THE COMMERCIALLY INSURED (2008)}

doi:10.1136/sextrans-2011-050108.244

G Janes, A Beard, G Tao, K Hoover, C Kent. Centers for Disease Control and Prevention, Atlanta, USA

Background Perinatal transmission of sexually transmitted infections is a preventable source of morbidity. In the USA, the incidence 
of congenital syphilis has increased by $23 \%$ since 2005 and, in 2008, 141 infants were infected with HIV, despite the fact that the CDC recommends routine prenatal syphilis and HIV screening. To encourage compliance with guidelines, many payers rely on claims data to track utilisation. In this study, the percentage of women screened for syphilis or HIV during their pregnancy was estimated using MarketScan claims data, as a measure of compliance and of the feasibility of using these data for assessment of prenatal screening. Methods We analysed data from the MarketScan database, that allows linkage of de-identified patient and utilisation data from varying sites of care. The analysis was restricted to women who delivered a live birth in 2008, and who were continuously enrolled in one health plan for at least 293 days prior to delivery. The eligible population was identified using the coding algorithm for a live birth defined by HEDIS.

Results Among the 220571 women with a live birth in 2008, 87.0\% received a syphilis test during their pregnancy, $74.1 \%$ received an HIV test and $73.3 \%$ received both. The test rate for syphilis was highest for those 31-35 yrs (88.2\%) and lowest for those 15-20 yrs (78.3\%). The test rate for HIV was highest among those $21-25$ yrs $(75.2 \%)$ and lowest among those $15-20$ yrs (70\%). Both prenatal syphilis and HIV test rates were highest among those insured by a preferred provider plan. Regionally, testing rates were highest in the South $(89.8 \%$ for syphilis and $82.1 \%$ for HIV) and lowest in the Northeast for syphilis (82.8\%) and in the West for HIV (62.9\%).

Conclusions The results suggest fairly good compliance with guidelines for prenatal HIV and syphilis screening, although utilisation still varies by age, insurance type and geographic region. The MarketScan database represents individuals covered by employment-based health plans, which is how most Americans are insured. These data provide a unique opportunity to assess utilisation among the insured, at a time when the USA is poised to move a larger proportion of the population into this group. Tracking of yearto-year changes could help public health and healthcare organizations partner to improve care and target interventions to ensure equal access to these critical services.

\section{P1-S6.21 CHLAMYDIA TRACHOMATIS SCREENING INITIATIVE AMONG FEMALE US ARMY SOLDIERS DEPLOYED TO KOREA}

doi:10.1136/sextrans-2011-050108.245

\begin{abstract}
${ }^{1} \mathrm{~N}$ Jordan, ${ }^{1} \mathrm{~N}$ Clemmons, ${ }^{2} \mathrm{~J}$ Gaydos, ${ }^{3} \mathrm{~J}$ Fishman, ${ }^{3} \mathrm{~T}$ Jacobsmuhlen, ${ }^{3} \mathrm{H}$ Choon Lee, ${ }^{3}$ M Meyers, ${ }^{3}$ T Klein. ${ }^{1}$ US Army Public Health Command, Aberdeen Proving Grounds, Edgewood, USA; ${ }^{2}$ Armed Forces Health Surveillance Center, Silver Spring, USA; ${ }^{3}$ USA MEDDAC, Republic of South Korea
\end{abstract}

Objectives To determine the prevalence of Chlamydia trachomatis (Ct) infections among female US Army soldiers deployed to the Republic of Korea; to identify high-risk groups; and to develop data to inform Army leaders and public health decision makers regarding the program's impact.

Methods All females reporting (in-processing) to the Eighth US Army, Republic of Korea, between 1 November 2007 and 31 December 2009 received an educational STI brief and a questionnaire, and were requested to provide a urine sample for $\mathrm{Ct}$ and gonorrhoea testing using the Aptima Combo 2 Assay. Contact tracing was conducted for all identified cases. Descriptive analyses of the population of interest and data collected during calendar years 2008-2009 were performed. A logistic regression model was generated to identify statistically significant risk factors.

Results Women deployed to Korea differed somewhat demographically when compared to the overall female Army population; a higher proportion of known high risk groups was observed (eg, women under 25 years of age). A total of 3761 women were screened during the 2 calendar years, with an overall positive rate of $5.7 \%$. Ct rates were as high as $11.4 \%$ among women under 20 years, and decreased with increasing age. Rates varied considerably by race, with the highest rates observed among American Indians/Alaskan natives $(9.3 \%)$, followed by Black females (6.5\%). The Eighth US Army screening program was associated with diagnosis of infections at an earlier age (22.1 years on average during the screening program, as compared to 26.1 years on average before the program was initiated). Statistically significant differences were observed, with higher rates found among Black women and women $<25$ years of age.

Conclusion A high prevalence of infection was observed, highlighting the need for a continued Eighth US Army screening program. Furthermore, the program demonstrated that universal screening during Army in-processing was feasible and resulted in detection of $\mathrm{Ct}$ at earlier ages, which likely reduces disease transmission, medical complications, and associated costs with treatment. These findings have implications not only for the continuation of the program in the Republic of Korea, but also for the expansion of such programs during in-processing activities among other military high risk populations (eg, Army recruits)

\section{P1-S6.22 CHARACTERISTICS OF CHLAMYDIA TESTING BY A LARGE COMMERCIAL LABORATORY CORPORATION, USA, 2008-2010}

doi:10.1136/sextrans-2011-050108.246

${ }^{1} \mathrm{C}$ Kent, ${ }^{1} \mathrm{G}$ Tao, ${ }^{1} \mathrm{~K}$ Hoover, ${ }^{2} \mathrm{~B}$ Body, ${ }^{2} \mathrm{M}$ Nye. ${ }^{1} \mathrm{CDC}$, Atlanta, USA; ${ }^{2}$ Laboratory Corporation of America, USA

Background US guidelines recommend chlamydia screening for all sexually active young women, HIV-infected persons, and men who have sex with men (MSM). Several types of chlamydia tests are available, including nucleic amplification tests (NAATs), DNA probe, and culture. Different assays have different sensitivity and specificity, and sensitivity and specificity also varies by specimen type. Because reported chlamydia trends could be impacted by shifts in the proportion of tests and specimen types used by providers and laboratories, it is important to monitor changes in test usage to help interpret reported trends in morbidity. We examined chlamydia testing data from one of the two largest commercial laboratories in the USA

Methods Among chlamydia tests performed from June 2008-to July 2010 by this laboratory, we assessed the frequency of test types used by year, sex, specimen type, and positivity of these tests.

Results During these 2 years, 2.9 million specimens were tested for chlamydia. Among those tested, $86 \%$ were women. Tests performed were: $77 \%$ NAATs, $23 \%$ DNA probes, and $0.3 \%$ cultures. Between year 1 and 2, NAATs increased from 73\% to $80 \%$ of all tests, and DNA probes decreased from $27 \%$ to $19 \%$. Test type varied by sex; $88 \%$ of tests among men were NAATs compared to $75 \%$ among women. The top specimen types among women tested by NAAT were: $45 \%$ cervical, $35 \%$ urine and $18 \%$ vaginal. Chlamydia positivity varied by test type: $5.1 \%$ NAAT, $1.4 \%$ DNA probe, and $2.3 \%$ culture.

Conclusions NAATs were the most frequently used chlamydia test and their use increased over the evaluation period, creating a smaller market for other tests. Although vaginal swabs are the most sensitive specimen for testing women by NAAT, currently they are the least common specimen type used for testing. This may reflect the delayed introduction (FDA clearance) of the vaginal swab compared to other collection devices. Stocking the wide assortment of collection devices required for testing different specimens in different populations might also be a barrier for providers. Because 